\title{
Aquatic insects and their importance in assessing ecosystem health
}

\section{Editorial}

Insects are the most successful group in the animal kingdom in terms of both richness and abundance and thus are the largest and most diverse group of invertebrates. They outnumber all animal species and compete with plant and microbial species richness. Among insects, aquatic insects are specialized group which exhibit rich adoptability and hence one more diverse. Micro and macro habitat distribution of aquatic insect population is due to their varied life style such as benthic (associated with the bottom substrates), clinging (clinging to the substrate with grasping claws or disks), sprawling (crawl along the protected surfaces of the substrates), climbing (reside on aquatic plant stems and other shoreline substrates), burrowing (burrow into the soft bottom), floating and swimming (which are not associated with a substrate for attachment).

With over 100,000 species, the aquatic insects grouped into 12 orders have been reported to harbour freshwater ecosystem and are prominent among other aquatic fauna. Off these, orders such as Diptera, Coleoptera and Trichopteraare more diverse and constitutes $43 \%, 18 \%$ and $15 \%$, respectively. These insects devote one or more stages of their life cycle in water and involve in important of ecological function in the freshwaters ecosystem like processing organic matter, food for predators, transporting energy flow between different trophic level. With various functional feeding groups viz., shredders, scrapers, collectors-gathers, collectors-filter feeders and predators, aquatic insects are links in nutrient cycling inter connected and their biological interactions often have significant effects on community structure in the freshwater ecosystem. In addition to this ecosystem function, they are very good indicators of the anthropogenic impact of aquatic environment and hence, they are used in bio-monitoring methods in aquatic system. Further the economic importance of biodiversity and role of aquatic insects facilitate sustainable culture fisheries management and practice.

India is bestowed with diverse freshwater ecosystem like streams, rivers, ponds, wetlands, lakes and reservoirs which serve as shelters for several aquatic insects as well as other floral and faunal communities. However, studies on freshwater organisms especially insects have received only less attention in India when compared to rest of the developed countries. A comprehensive review of studies on aquatic insects are carried out in Tamil Nadu, Kerala, Karnataka, Andra Pradesh revealed their relationship with physico-chemical parameters of their habitats. Further these studies extrapolate the utility of aquatic insects in monitoring changes in water quality and aquatic life in freshwater ecosystems and to identify the sources of pollution are currently employed in assessing the biomagnification due to pesticides and heavy metals.

Aquatic insect are the diverse group of insect, which used for various toxicological studies. This insect indicate the pollution status of water bodies. Hence for any pesticide registration the test on aquatic insect needed. OECD has given various guidelines to conduct the studies on pesticides in Daphnia sp., Chironomus immobilisation test. So insects play a important role for not only diversity but as a indicator of any pollutant in any water bodies. In water oxygen level
Volume 6 Issue 4 - 202 I

Kathirvelu Baskar, Shweta Gawade
Department of Ecotoxicology, Ross Lifescience Pvt., Ltd., India

Correspondence: Kathirvelu Baskar, Department of Ecotoxicology, Ross Lifescience Pvt., Ltd., Pune, Maharastra 4I 1026, India, Email suribaskar@hotmail.com

Received: July 14, 202I | Published: July 20, 2021

decreased, increased chironomous larvae, temperature increased decline of stonefly, pesticides increased affect the diversity and nutrient content increased population of some insect increased and found imbalance of diversity. The $\mathrm{pH}$, electric conductivity and Dissolved oxygen were directly proportional to insect population increased. Human contaminations were indicated by aquatic insects. The bacterial growth in insect indicates that elevated concentration of the phosphate and nitrate in the wetland ecosystem. The abundance of Gerris spinolae population indicates that the aquatic ecosystem was unpolluted. The bioindicator is better indicator than chemical indicator, because we cannot measure all the contaminated chemicals in the ecosystem. The aquatic insect population directly proportional to seasonal variations.

The aquatic ecosystems being a rich source of water availability are mostly targeted by people for fulfilling their needs. Further, in the ecosystem is threatened due to forest fragmentation, expansion of agriculture, construction of dams and mining greatly affects the vegetation area and the faunal diversity associated with in it. In recent days, uncontrolled and unregulated human activities have extensively transformed natural ecosystems into various other landforms, especially commercial plantations, greatly depleting biomass and diversity levels in areas remaining under forest cover. Because of their distribution in varied microhabitats, high abundance, high fecundity, low generation time and rapid colonization, aquatic insects are best tools in analysing the structure and function of fresh water ecosystem. Hence, it is inevitable that more researches needed in the direction of assessing the health of fresh water ecosystems using aquatic insects prior to planning conservation and management strategies.

\section{Acknowledgments}

None. 


\section{Funding}

None.

\section{Conflicts of interest}

The authors declare that there is no conflict of interest. 